\title{
Higher glucose levels and prevalence of prediabetes in patients with autoimmune thyroiditis
}

Georgios Boutzios, Krystallenia Alexandraki, Stavros Liatis, Eleni Lampropoulou, Georgios Nikolopoulos, Aikaterini Bouki, Eleni Papaoiconomou, Kokkona Paradeisi, Nikolaos Tentolouris, Gregory Kaltsas

Section of Endocrinology, Department of Pathophysiology, National and Kapodestrian University of Athens Medical School, Athens, Greece

\section{Introduction}

Thyroid autoimmunity and DM2 are the commonest endocrine disorders in the general population.

\section{Aim of the study}

The aim of the study was to investigate a possible association between thyroid autoimmunity and $\beta$-cell secretion in patients with impaired fasting glucose and/ or impaired glucose tolerance.

\section{Methods}

604 patients (457 females) $46.5 \pm 14.2$ years old with impaired fasting glucose (IFG) and/or impaired glucose tolerance (IGT). The population was divided in 2 subgroups according to the presence of autoimmune thyroiditis (AIT, TgAb and/or TPOAb positive) with normal TSH or not (non-AIT).

Characteristics such as age, gender, waist circumference $(\mathrm{cm})$, BMI were analysed.

291 patients underwent an OGTT after oral administration of $75 \mathrm{gr}$ glucose. Glucose (mg/dl) and insulin $(\mu \mathrm{IU} / \mathrm{mL})$ at 5 different times (0-30-60-90-120 min), $1^{\text {st }}$ and $2^{\text {nd }}$ phase of insulin sensitivity index (ISI) and ISI were assessed (from the rest patients only fasting glucose and insulin were measured).

Glycated hemoglobin (HbAlc), HOMA and QUICKI insulinresistance (IR) indices, and IR status as HOMA $>2.16$ and OUICKI $<0.34$ were also assessed.
Exclusion criteria: Patients with hypothyroidism or diabetes mellitus DM2 were excluded from the study.

\section{Results}

Patients with AIT $(\mathrm{N}=311)$ were older compared to the non-AIT $(\mathrm{N}=293)[49.2 \pm 13.9$ vs. $44.2 \pm 14.7$ $(\mathrm{p}<0.001)]$, had higher glucose levels $[99.9 \pm 12$ vs. $95.5 \pm 12.5$ $(p<0.001)]$, and higher rates of prediabetes: $71,2 \%$ (IFG and/or IGT) $($ chi-square $=27.2, \mathrm{p}<0.001)$ but similar rates of IR (44.6\%) (chi-square $=21.5, \mathrm{p}<0.001$ ).

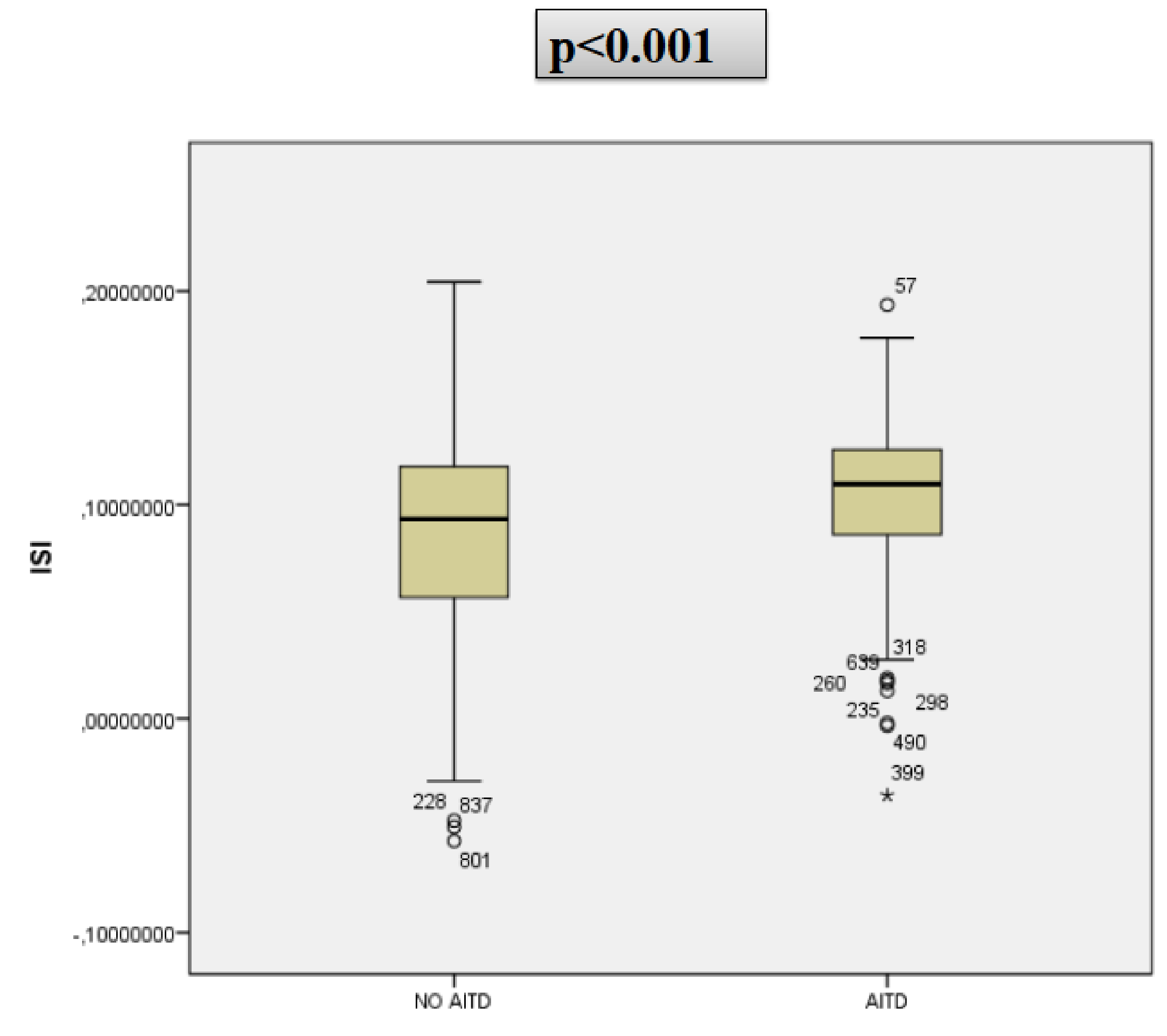

IN PREDIABETIC PATIENTS ONLY

Prediabetic patients with AIT who underwent OGTT $(\mathrm{N}=221)$ had higher $1^{\text {st }}$ phase of ISI $(1470.9 \pm 458.2)$ and ISI $(0.1 \pm 0.03)$ compared to those without AIT ( $1^{\text {st }}$ phase of ISI: $1262.8 \pm 596.4, \quad \mathrm{p}<0.001 ; \quad$ ISI: $0.09 \pm 0.05, \mathrm{p}<0.001)$.

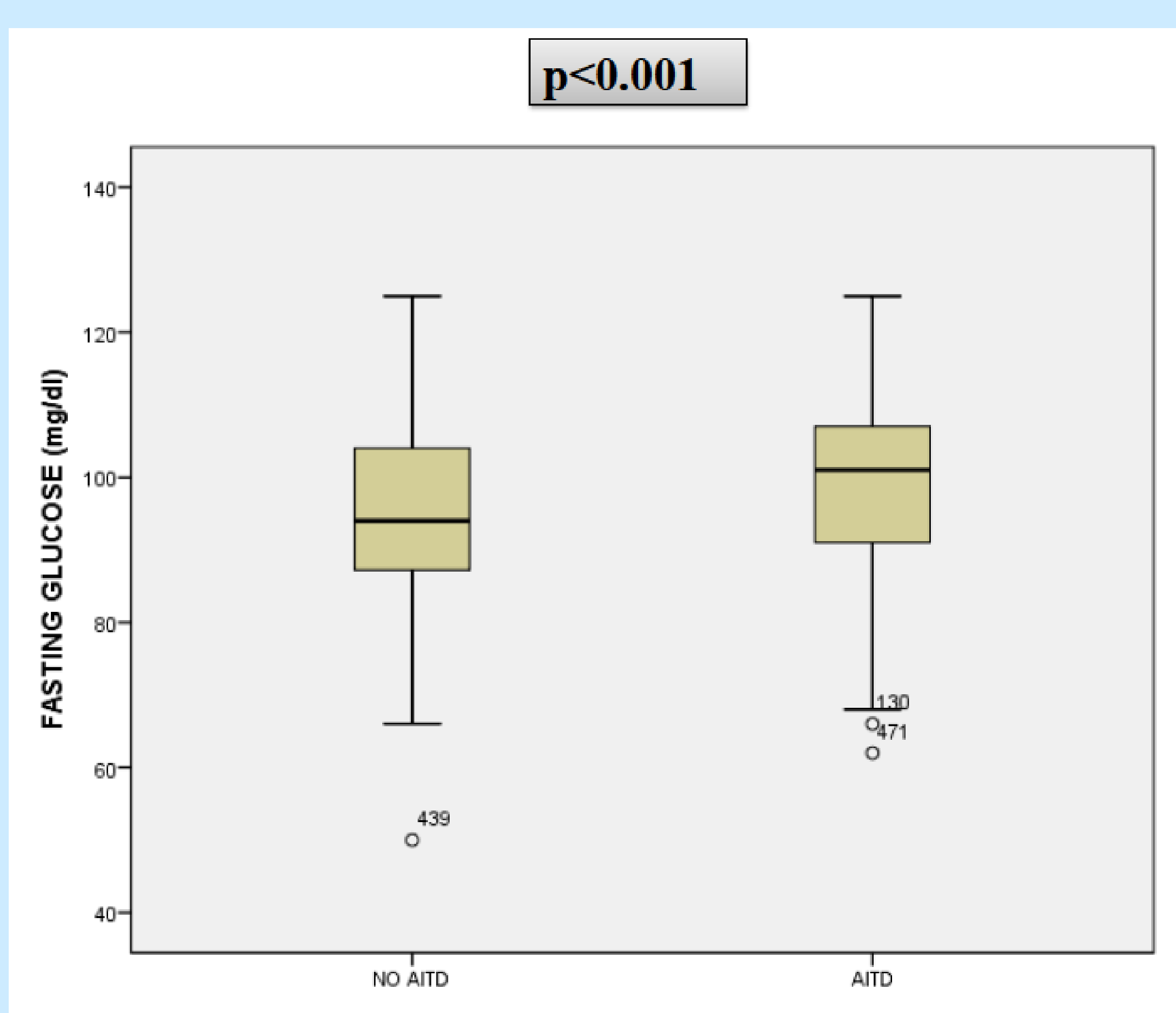

IN ALL THE POPULATION STUDIED

\section{Conclusion}

- Patients with AIT and impaired $\beta$-cell secretion had higher glucose levels with similar rate of insulin resistance and higher rates of prediabetes compared to non-AIT.

- Thyroid autoimmunity could be eventually a possible factor modifying $\beta$-cell secretion. Nevertheless further studies are needed to confirm these findings.

References

1. Hashimoto SM et al. Autoimmunity does not contribute to the highly prevalent glucose metabolism disturbances in a Japanese Brazilian population. Ethn Dis. 2007

2. Tamayo $T$ et al. Diabetes in Europe: an update. Diabetes Res Clin Pract. 2014

3. Kasagi $\mathrm{K}$ et al. Thyroid function in Japanese adults as assessed by a general health check up system in relation with thyroid-related antibodies and other clinical parameters. Thyroid 2009 